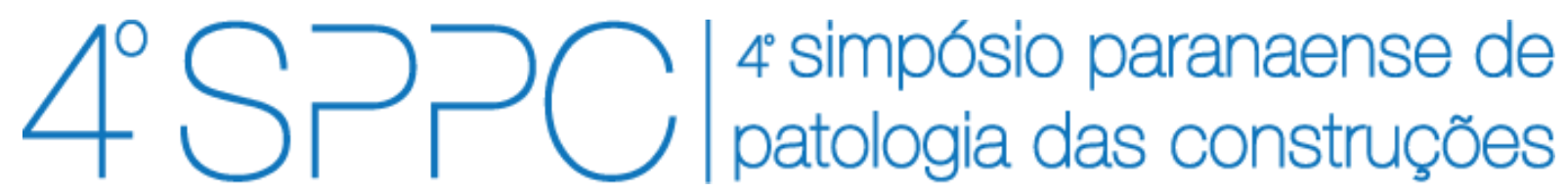

ISSN 2526-7248 artigo n. 4SPPC147,pp. 420-428, 2019

\title{
Método GUT para priorização na resolução das manifestações patológicas em edifícios residenciais na orla de Boa Viagem
}

\author{
Santos, Manueli Suêni da Costa1; Nunes, Allan Caetano Lucena²; Nascimento, \\ Carlos Fernando Gomes ${ }^{3}$; Silva, Cristiane Santana da ${ }^{4}$; Silva, Thaís Marques ${ }^{5}$; \\ Monteiro, Eliana Cristina Barreto ${ }^{5}$ \\ ${ }^{1}$ Mestranda em Engenharia Civil, Universidade Católica de Pernambuco, \\ manuelisueni@hotmail.com \\ ²Engenheiro Civil, Universidade Católica de Pernambuco, \\ allan.engcivil10@gmail.com \\ ${ }^{3}$ Graduando em Engenharia Civil, Universidade Católica de Pernambuco, \\ carlosfernando.gn@gmail.com \\ ${ }^{4}$ Mestranda em Engenharia Civil, Universidade Católica de Pernambuco, \\ cristiane_santana@msn.com \\ ${ }^{5}$ Mestranda em Engenharia Civil, Universidade de Pernambuco, \\ thaism21@ymail.com \\ ${ }^{6}$ Professora Doutora, Universidade Católica de Pernambuco/Universidade de \\ Pernambuco, eliana@poli.br
}

Resumo: O método GUT é uma ferramenta útil para priorizar as ações corretivas de forma racional, considerando a gravidade, urgência e tendência. Este trabalho objetiva criar uma matriz de priorização para as manifestações patológicas encontradas em oito edifícios residenciais. Foram realizadas visitas técnicas para identificar os problemas e compor a matriz. Corrosão de armaduras e descolamento de placas cerâmicas apresentaram maior pontuação na matriz de priorização. Conclui-se que o método GUT é eficaz para a tomada de decisão no ramo da construção civil, contribuindo para priorizar as soluções necessárias para correção das manifestações patológicas.

Palavras-chave: Método GUT, manifestações patológicas, matriz de priorização.

Abstract: The GUT method is a useful tool to prioritize corrective actions in a rational way, considering gravity, urgency and trend. This work aims to create a prioritization matrix for the pathological manifestations found in eight residential buildings. Technical visits were made to identify the problems and compose the matrix. Corrosion of reinforcement and detachment of ceramic plates presented higher scores in the prioritization matrix. It is concluded that the GUT method is effective for decision-making in the construction industry, contributing to prioritize the solutions needed to correct the pathological manifestations.

Keywords: GUT method, pathological manifestations, prioritization matrix. 
SANTOS, M.S.C.; NUNES, A.C.L.; NASCIMENTO, C.F.G.; SILVA, C.S.; SILVA, T.M.; MONTEIRO, E.C.B. MÉTODO GUT PARA PRIORIZAÇÃO NA RESOLUÇÃO DAS MANIFESTAÇÕES PATOLÓGICAS EM EDIFÍCIOS RESIDENCIAIS NA ORLA DE BOA VIAGEM. $4^{\circ}$ Simpósio Paranaense de Patologia das Construções (40 SPPC), artigo 4SPPC147, pp. 420 - 428, 2019.

\section{Introdução} DOI: $10.4322 / 2526-7248.064$

De acordo com Helene [1], "Patologia pode ser entendida como a parte da engenharia que estuda os sintomas, os mecanismos, as causas e origens dos defeitos das construções civis, ou seja, é o estudo das partes que compõem o diagnóstico do problema".

Grande parte das manifestações patológicas nas edificações apresenta sintomas característicos que possibilitam a identificação de sua origem, como também suas possíveis consequências. Edificações em zona litorânea estão sujeitas a agressividade do meio ambiente em condições ainda mais severas. Existem diversas manifestações patológicas encontradas em edificações residenciais, entre as quais se destacam as fissuras, manchas, eflorescências, corrosão de armadura e deterioração da estrutura de concreto armado.

Estes problemas podem ocorrer muito antes do previsto em projeto fazendo com que a estrutura tenha a sua durabilidade reduzida, como também diminuindo sua resistência. Estas manifestações podem ter origem em qualquer etapa no processo construtivo, observando-se, assim, a necessidade de manutenções preventivas, do controle tecnológico dos materiais empregados, de uma padronização e qualidade na execução dos projetos e da qualidade dos processos de execução.

De acordo com Nascimento, Santos e Almeida [2], o custo elevado para a resolução dos problemas e a falta de recursos faz com que estes problemas não sejam corrigidos de forma integral, sendo necessária uma ordem de priorização na resolução destas manifestações patológicas tendo como referência critérios como gravidade, urgência e tendência.

O presente trabalho se dispõe a criar uma ordem de priorização para resolução das manifestações patológicas encontradas em edificações residenciais na Orla da Praia de Boa Viagem na cidade do Recife a partir do método GUT (gravidade, urgência e tendência).

\section{Manifestações patológicas}

\subsection{Fissuras}

De acordo com Lapa [3], as trincas e fissuras são fenômenos próprios e inevitáveis do concreto armado e que podem se manifestar em cada uma das três fases de sua vida: fase plástica, fase de endurecimento e fase de concreto endurecido. Na fase plástica podem surgir trincas em virtude da retração plástica e do assentamento plástico; na fase de endurecimento, em virtude de restrições à precoce movimentação térmica, à precoce retração do endurecimento e ao assentamento diferencial dos apoios; na fase de concreto endurecido, as principais causas do aparecimento das trincas e fissuras são o subdimensionamento, o detalhamento inadequado, a construção sem os cuidados indispensáveis, as cargas excessivas, o ataque de sulfatos ao cimento do concreto, a corrosão das armaduras devida ao ataque de cloretos, a carbonatação e a reação álcali- agregado. 
SANTOS, M.S.C.; NUNES, A.C.L.; NASCIMENTO, C.F.G.; SILVA, C.S.; SILVA, T.M.; MONTEIRO, E.C.B. MÉTODO GUT PARA PRIORIZAÇÃO NA RESOLUÇÃO DAS MANIFESTAÇÕES PATOLÓGICAS EM EDIFÍCIOS RESIDENCIAIS NA ORLA DE BOA VIAGEM. $4^{\circ}$ Simpósio Paranaense de Patologia das Construções (40 SPPC), artigo 4SPPC147, pp. 420 - 428, 2019.

\subsection{Corrosão de armaduras} DOI: $10.4322 / 2526-7248.064$

Cascudo [4] define corrosão de elementos metálicos como sendo alterações de um metal de íon metálico pela sua alteração química ou eletroquímica com o meio ambiente.

Segundo Andrade [5], a corrosão das armaduras é caracterizada por ser um processo eletroquímico gerador de óxidos e hidróxidos de ferro denominados produtos de corrosão, que ocupam um volume significativamente superior ao volume original das barras metálicas. Por isso o mecanismo de corrosão do aço, no concreto, só se desenvolve em presença de água ou ambiente de umidade relativa elevada (U.R.> 60\%).

\subsection{Eflorescência}

Segundo Santos [6], as eflorescências são manchas de coloração normalmente branca que surgem no concreto devido ao acúmulo de solução saturada de hidróxido de cálcio na superfície do concreto, podendo gerar estalactites nas zonas de maior porosidade do concreto.

Silva [7] diz que na eflorescência ocorre a formação de depósitos salinos na superfície do concreto, resultante da água de infiltrações ou intempéries. Esses sais constituintes podem ser agressivos e também pode causar desagregação profunda, além da modificação do aspecto visual na estrutura, pois há um contraste de cor entre os sais e o substrato sobre os quais se depositam.

\section{Método GUT}

De acordo com Martins [8], o método GUT (Gravidade, Urgência e Tendência) é uma ferramenta desenvolvida por Charles $\mathrm{H}$. Kepner e Benjamin B. Tregoe com o intuito de definir prioridades de forma racional. Tal método consiste em analisar a gravidade ou impacto do problema nas operações e pessoas envolvidas, a urgência a brevidade necessária para a resolução dos problemas e a tendência ou apresentação de melhora ou piora do problema. Nele se utiliza de três escalas para definir uma prioridade, e através da combinação delas é possível fazer uma priorização eficaz, orientando a tomada de decisão e a resolução de problemas.

Cada problema a ser analisado recebe uma nota de 1 a 5 em cada uma das características: gravidade, urgência e tendência. Assim os pontos da escala GUT atribuídos a cada um dos problemas são multiplicados, dando origem a um valor resultante para cada problema. Desta forma, as tomadas de decisões para os problemas podem ser gerenciadas de acordo com os valores máximos obtidos.

O Quadro 1 apresenta as pontuações adotadas para compor a matriz de priorização do método GUT. 
SANTOS, M.S.C.; NUNES, A.C.L.; NASCIMENTO, C.F.G.; SILVA, C.S.; SILVA, T.M.; MONTEIRO, E.C.B. MÉTODO GUT PARA PRIORIZAÇÃO NA RESOLUÇÃO DAS MANIFESTAÇÕES PATOLÓGICAS EM EDIFÍCIOS RESIDENCIAIS NA ORLA DE BOA VIAGEM. $4^{\circ}$ Simpósio Paranaense de Patologia das Construções (40 SPPC), artigo 4SPPC147, pp. 420 - 428, 2019. DOI: $10.4322 / 2526-7248.064$

Quadro 1: Pontuação pelo método GUT (Oliveira, 1995 apud Martins, 2017)

\begin{tabular}{|c|c|c|c|}
\hline Pontos & Gravidade & Urgência & Tendência \\
\hline $\mathbf{5}$ & $\begin{array}{c}\text { Consequência se } \\
\text { nada for feito }\end{array}$ & $\begin{array}{c}\text { Prazo para } \\
\text { tomada de } \\
\text { decisão }\end{array}$ & $\begin{array}{c}\text { Proporção do } \\
\text { problema no } \\
\text { futuro }\end{array}$ \\
\hline $\mathbf{E}$ & $\begin{array}{c}\text { Prejuízos } \\
\text { graves }\end{array}$ & $\begin{array}{c}\text { É necessário ação } \\
\text { imediata }\end{array}$ & $\begin{array}{c}\text { Se nada for feito, } \\
\text { agravamento } \\
\text { imediato }\end{array}$ \\
\hline $\mathbf{4}$ & Muito graves & $\begin{array}{c}\text { Com alguma } \\
\text { urgência }\end{array}$ & $\begin{array}{c}\text { Vai piorar a curto } \\
\text { prazo }\end{array}$ \\
\hline $\mathbf{3}$ & Graves & $\begin{array}{c}\text { O mais cedo } \\
\text { possível }\end{array}$ & $\begin{array}{c}\text { Vai piorar a médio } \\
\text { prazo }\end{array}$ \\
\hline $\mathbf{2}$ & Pouco graves & $\begin{array}{c}\text { Pode esperar um } \\
\text { pouco }\end{array}$ & $\begin{array}{c}\text { Vai piorar a longo } \\
\text { prazo }\end{array}$ \\
\hline $\mathbf{1}$ & Sem gravidade & Não tem pressa & Não vai piorar \\
\hline
\end{tabular}

\section{Metodologia}

O desenvolvimento do estudo de caso se deu através da inspeção visual das manifestações patológicas, utilizando como material de auxílio de uma câmera fotográfica para registrar os problemas encontrados nas edificações e de uma planilha para a coleta de dados relevantes sobre as edificações. A vistoria foi realizada em 4 (quatro) edifícios na orla da praia de Boa Viagem, em Recife, Pernambuco.

O ambiente onde estão inseridos os imóveis utilizados neste estudo é predominantemente marítimo e urbano. A presença do gás carbônico é constante nessas edificações, pelo fato de estarem localizadas em uma avenida bastante movimentada como é o caso da Avenida Boa Viagem.

Com a identificação destes problemas, torna-se possível criar as matrizes de priorização para resolução destas manifestações patológicas, através da aplicação do método GUT, considerando a gravidade, urgência e tendência de cada manifestação patológica. 
SANTOS, M.S.C.; NUNES, A.C.L.; NASCIMENTO, C.F.G.; SILVA, C.S.; SILVA, T.M.; MONTEIRO, E.C.B. MÉTODO GUT PARA PRIORIZAÇÃO NA RESOLUÇÃO DAS MANIFESTAÇÕES PATOLÓGICAS EM EDIFÍCIOS RESIDENCIAIS NA ORLA DE BOA VIAGEM. $4^{\circ}$ Simpósio Paranaense de Patologia das Construções (40 SPPC), artigo 4SPPC147, pp. 420 - 428, 2019.

\section{Resultados e discussões} DOI: $10.4322 / 2526-7248.064$

\subsection{Edifício 1}

O edifício 1 é residencial, apresenta 9 pavimentos tipo, 1 pavimento térreo e 1 pavimento no subsolo utilizado para garagem.

Durante a visita ao edifício, foram encontradas diversas manifestações patológicas. A falta de manutenção periódica ou a manutenção inadequada agrava ainda mais a situação do edifício e o torna mais vulnerável para a origem de novos problemas. A seguir, nas Figura 1, é apresentada uma viga com presença de corrosão de armaduras.

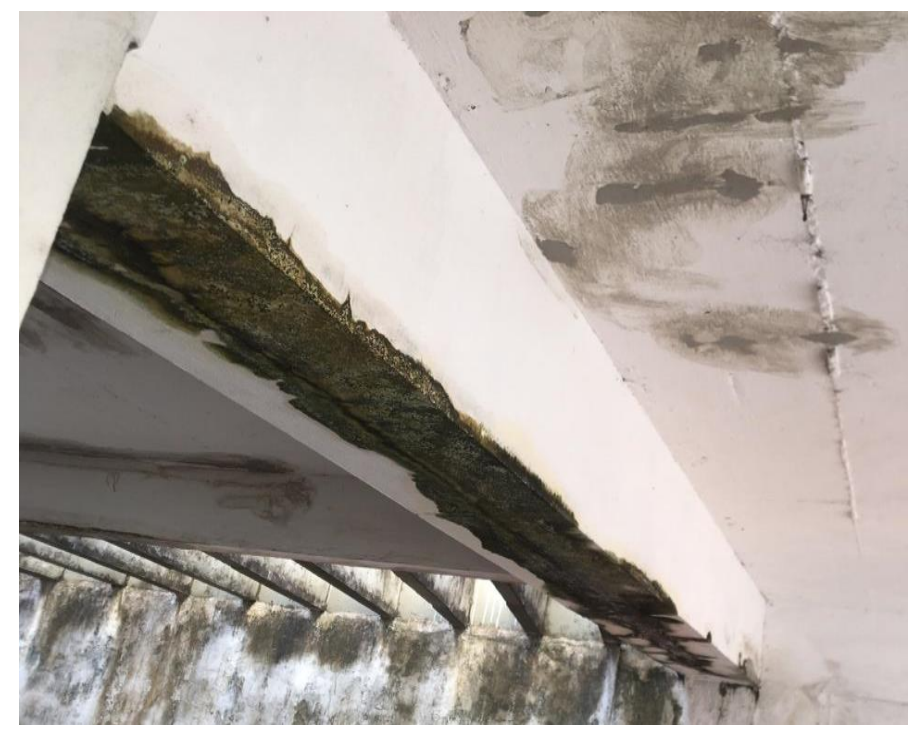

Figura 1: Viga em processo de corrosão no Edifício 01

As principais manifestações encontradas nesta edificação foram corrosão de armaduras, presença de mofo e o descolamento de placas cerâmicas e trincas. A partir destes registros fotográficos foi possível criar a matriz de priorização GUT, conforme apresenta a Tabela 1.

Tabela 1: Matriz de priorização GUT do Edifício 1

\begin{tabular}{c|c|c|c|c}
\hline Manifestação patológica & $\mathbf{G}$ & $\mathbf{U}$ & $\mathbf{T}$ & Pontuação \\
\hline Corrosão de armaduras & 4 & 4 & 3 & 48 \\
\hline Descolamento de placas cerâmicas & 3 & 3 & 2 & 18 \\
\hline Trincas & 2 & 3 & 2 & 12 \\
\hline Mofo & 2 & 2 & 3 & 12 \\
\hline
\end{tabular}

A corrosão de armaduras apresenta 48 pontos, ou seja, a maior pontuação na matriz, e consequentemente, deve ser priorizada na resolução dos problemas. Em seguida, aparece o descolamento de placas cerâmicas com 18 pontos, trincas com 12 pontos e mofo com 12 pontos. 


\subsection{Edifício 2}

As manifestações patológicas encontradas na edificação foram: corrosão de armaduras, eflorescência (Figura 2), fissuras, descolamento de placa cerâmica, descolamento de argamassa e bolor e mofo.

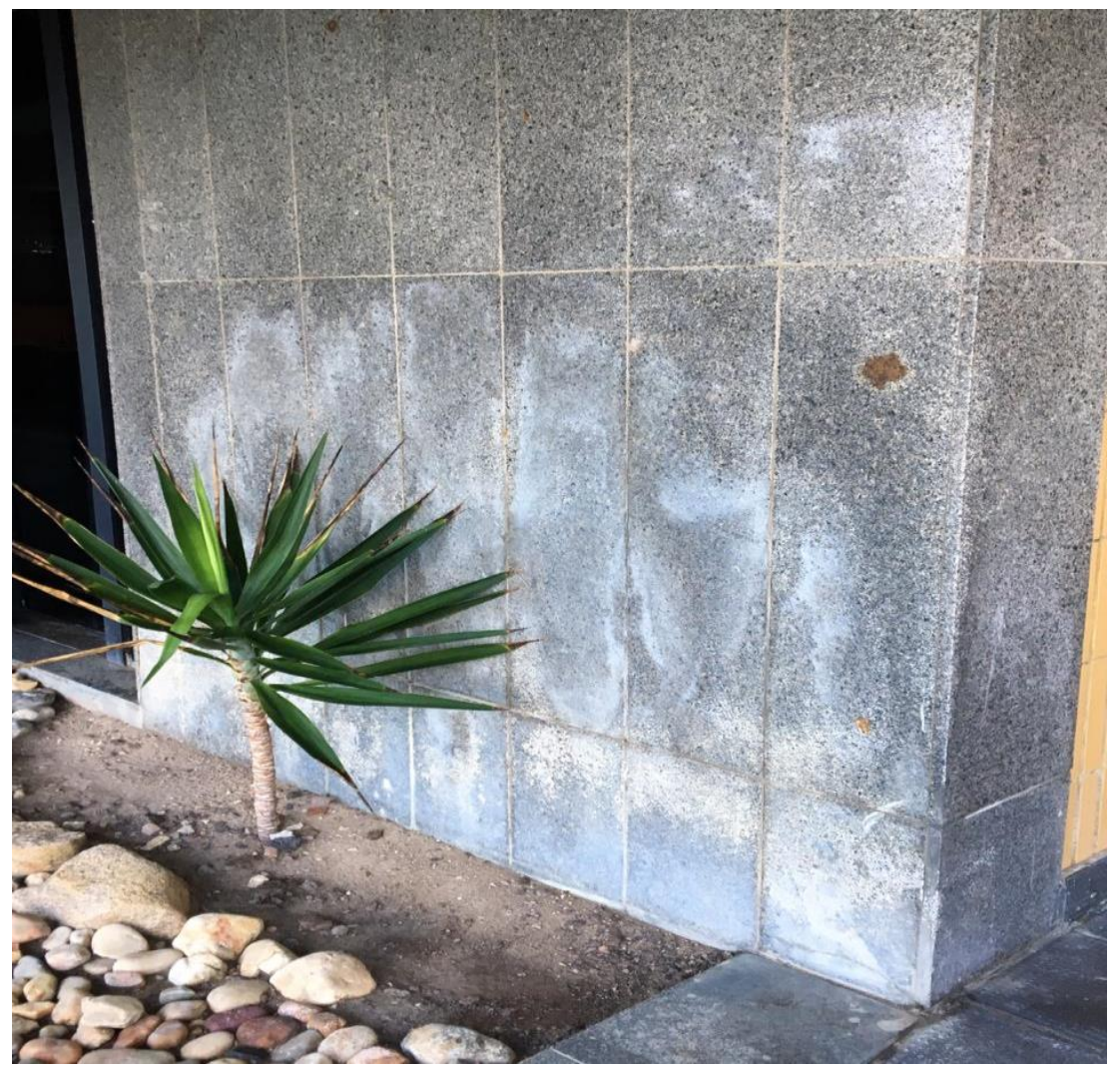

Figura 2: Eflorescência identificada em Edifício 2

A Tabela 2 apresenta a matriz de priorização GUT para o edifício 2.

Tabela 2: Matriz de priorização GUT do Edifício 2.

\begin{tabular}{c|c|c|c|c}
\hline Manifestação patológica & G & U & T & Pontuação \\
\hline Corrosão de armaduras & 3 & 3 & 3 & 27 \\
\hline Descolamento de placas cerâmicas & 3 & 3 & 2 & 18 \\
\hline Eflorescência & 2 & 2 & 3 & 12 \\
\hline Fissuras & 2 & 2 & 2 & 8 \\
\hline $\begin{array}{c}\text { Descolamento em revestimento de } \\
\text { argamassa por empolamento }\end{array}$ & 2 & 1 & 3 & 6 \\
\hline Bolor e mofo & 2 & 1 & 2 & 4 \\
\hline
\end{tabular}

A corrosão de armaduras apresenta 27 pontos. Em seguida, aparece 0 descolamento de placas cerâmicas com 18 pontos, eflorescência com 12 pontos, fissuras com 8 pontos, e descolamento em revestimento de argamassa por empolamento e bolor e mofo, respectivamente com 6 e 4 pontos. 


\subsection{Edifício 3}

Neste edifício, foi encontrado um número menor de manifestações patológicas comparando-se aos demais. A realização de manutenções periódicas assegura ao edifício uma preservação em sua estrutura e combate o surgimento de novas manifestações patológicas.

As principais manifestações encontradas nesta edificação foi a corrosão de armaduras e fissuras (Figura 3).

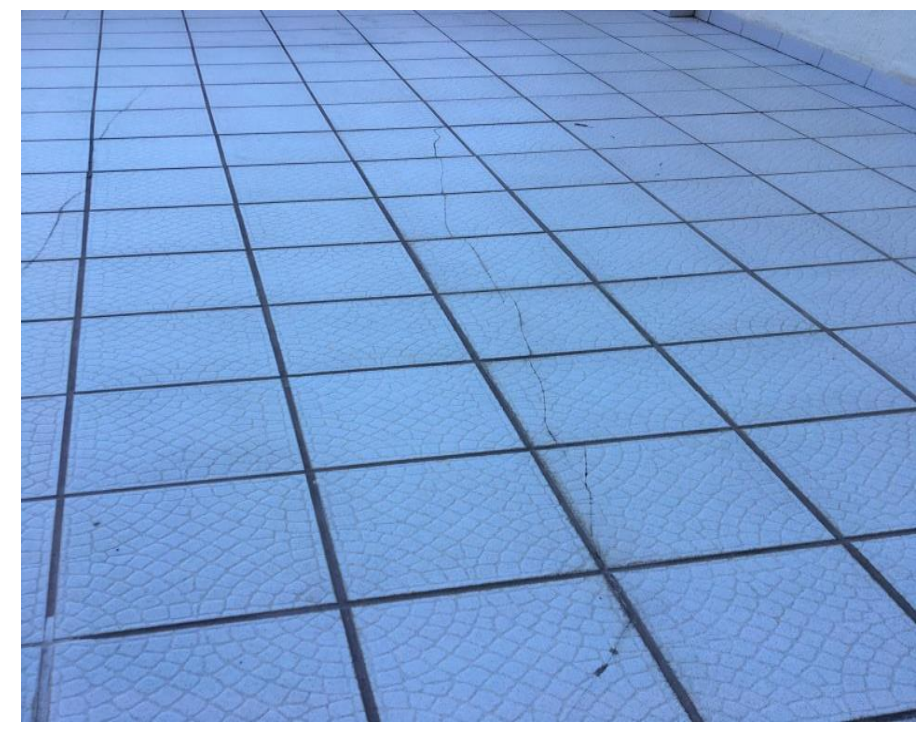

Figura 3: Fissuras no piso cerâmico do Edifício 3

A partir da inspeção visual, foi possível criar a matriz de priorização GUT, conforme apresenta a Tabela 3.

Tabela 3: Matriz de priorização GUT do Edifício 3

\begin{tabular}{c|c|c|c|c}
\hline Manifestação patológica & G & U & T & Pontuação \\
\hline Corrosão de armaduras & 3 & 3 & 3 & 27 \\
\hline Fissuras & 2 & 2 & 2 & 8 \\
\hline
\end{tabular}

A corrosão de armaduras apresenta 27 pontos e deve ser priorizada na resolução dos problemas. Em seguida, aparecem as fissuras com 8 pontos.

\subsection{Edifício 4}

Devido ao tempo, exposição a ambientes agressivos e falta de inspeção periódica, a vida útil do edifício pode ser reduzida, aparecendo sinais de manifestações patológicas na estrutura. 
SANTOS, M.S.C.; NUNES, A.C.L.; NASCIMENTO, C.F.G.; SILVA, C.S.; SILVA, T.M.; MONTEIRO, E.C.B. MÉTODO GUT PARA PRIORIZAÇÃO NA RESOLUÇÃO DAS MANIFESTAÇÕES PATOLÓGICAS EM EDIFÍCIOS RESIDENCIAIS NA ORLA DE BOA VIAGEM. $4^{\circ}$ Simpósio Paranaense de Patologia das Construções (40 SPPC), artigo 4SPPC147, pp. 420 - 428, 2019.

DOI: $10.4322 / 2526-7248.064$

As principais manifestações encontradas nesta edificação foram corrosão de armaduras, eflorescência (Figura 4) e a presença de mofo e trincas.

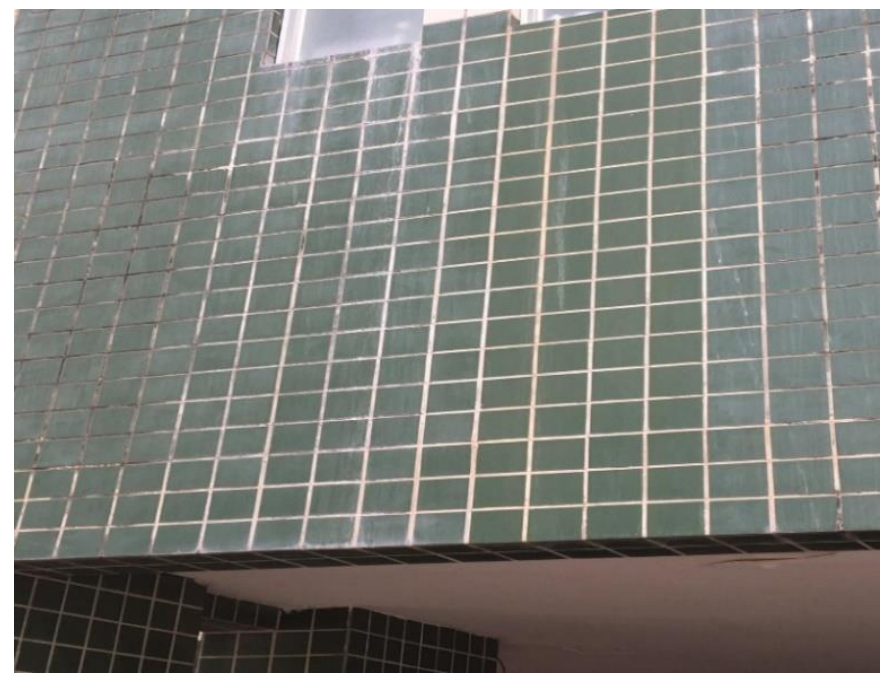

Figura 4: Eflorescência na fachada lateral do Edifício 4

A partir do registro fotográficos foi possível criar a matriz de priorização GUT, conforme apresenta a Tabela 4.

Tabela 4: Matriz de priorização GUT do Edifício 4

\begin{tabular}{c|c|c|c|c}
\hline Manifestação patológica & $\mathbf{G}$ & $\mathbf{U}$ & $\mathbf{T}$ & Pontuação \\
\hline Corrosão de armaduras & 4 & 3 & 3 & 36 \\
\hline Eflorescência & 2 & 2 & 3 & 12 \\
\hline Trincas & 3 & 3 & 2 & 18 \\
\hline Bolor e mofo & 2 & 1 & 2 & 4 \\
\hline
\end{tabular}

A corrosão de armaduras apresenta 36 pontos. Em seguida, aparecem trincas com 18 pontos, eflorescência com 12 pontos e a presença de bolor e mofo com 4 pontos.

\section{Considerações finais}

Diante dos estudos realizados e resultados obtidos, observa-se a presença de um elevado número de anomalias na área dos estudos. Entre elas, destaca-se a corrosão de armadura, deslocamento de placas cerâmicas, fissuras, trincas, eflorescência, bolor e mofo.

A priorização por meio do método GUT sugere que, nos locais estudados, os recursos destinados à manutenção sejam aplicados nas correções dos elementos estruturais que possuem armaduras em estado de corrosão, seguidos daqueles com menor pontuação na matriz de priorização como a presença de bolor e mofo. 
SANTOS, M.S.C.; NUNES, A.C.L.; NASCIMENTO, C.F.G.; SILVA, C.S.; SILVA, T.M.; MONTEIRO, E.C.B. MÉTODO GUT PARA PRIORIZAÇÃO NA RESOLUÇÃO DAS MANIFESTAÇÕES PATOLÓGICAS EM EDIFÍCIOS RESIDENCIAIS NA ORLA DE BOA VIAGEM. $4^{\circ}$ Simpósio Paranaense de Patologia das Construções (40 SPPC), artigo 4SPPC147, pp. 420 - 428, 2019. DOI: $10.4322 / 2526-7248.064$

Conclui-se que a ferramenta GUT pode ser aplicada como facilitadora do planejamento das atividades de manutenção em edificações de concreto armado, com base nos resultados e na avaliação proposta.

Em relação à utilização do método GUT para o estabelecimento da ordem de prioridade de resolução entre os problemas encontrados, foi comprovado a sua aplicabilidade individual nas áreas de estudo, com base na possível realização da hierarquização de riscos referentes às manifestações patológicas avaliadas.

\section{Referências}

[1] Helene, Paulo R. L. Manual para Reparo, Reforço e Proteção das Estruturas de Concreto. 2 ed. São Paulo: Pini, 1992.

[2] Nascimento, Carlos; SANTOS, Manueli; ALMEIDA, Maria. Método GUT (gravidade, urgência e tendência) e método dos fatores para priorização na resolução de manifestações patológicas e estimativa de vida útil de elementos em edifício na cidade do Recife. In: CONGRESSO BRASILEIRO DE PATOLOGIA DAS CONSTRUÇÕES, 3., 2018, Campo Grande. Anais do Congresso Brasileiro de Patologia das Construções. Campo Grande: Anais Cbpat, 2018. p. 1493 - 1502.

[3] Lapa, José Silva. Patologia, recuperação e reparo das estruturas de concreto. 2008. 56 f. TCC (Graduação) - Curso de Construção Civil, Universidade Federal de Minas Gerais, Belo Horizonte, 2008.

[4] Cascudo, O. O Controle da corrosão de armaduras em concreto: inspeção e técnicas eletroquímicas. 1 ed. São Paulo: Pini, 1997.

[5] Andrade, Jairo José de Oliveira. Durabilidade das estruturas de concreto armado: análise das manifestações patológicas nas estruturas. 1997. $151 \mathrm{f}$. Dissertação (Mestrado) - Curso de Engenharia Civil, Universidade Federal do Rio Grande do Sul, Porto Alegre, 1997.

[6] Santos, Camila Freitas dos. Patologia de estruturas de concreto armado. 2014. 91 f. TCC (Graduação) - Curso de Engenharia Civil, Universidade Federal de Santa Maria, Santa Maria, 2014.

[7] Silva, Luiza Kilvia da. Levantamento de manifestações patológicas em estruturas de concreto armado no estado do Ceará. 2011. 61 f. TCC (Graduação) - Curso de Engenharia Civil, Universidade Federal do Ceará, Fortaleza, 2011.

[8] Martins, N. et al. Priorização na Resolução de Manifestações Patológicas em Estruturas de Concreto Armado: Método GUT. Revista de Engenharia e Pesquisa Aplicada, Recife, v. 1, 2017. 